\title{
Fusion of neutron-rich oxygen nuclei
}

\author{
Romualdo deSouza ${ }^{1,2, \star}$, Justin Vadas ${ }^{1,2}$, Varinderjit Singh ${ }^{1,2}$, Blake Wiggins ${ }^{1,2}$, Tracy Steinbach ${ }^{1,2}$, Zidu Lin $^{2,3}$, \\ Chuck Horowitz ${ }^{2,3}$, Lagy Baby ${ }^{4}$, Sean Kuvin ${ }^{4}$, Vandana Tripathi ${ }^{4}$, Ingo Wiedenhover ${ }^{4}$, and Sait Umar ${ }^{5}$ \\ ${ }^{1}$ Department of Chemistry, Indiana University, Bloomington, IN 47405 USA \\ ${ }^{2}$ Center for Exploration of Energy and Matter, Indiana University, Bloomington, IN 47408 USA \\ ${ }^{3}$ Department of Physics, Indiana University, Bloomington, IN 47405 USA \\ ${ }^{4}$ Department of Physics, Florida State University, Tallahassee, FL, 32306 USA \\ ${ }^{5}$ Department of Physics and Astronomy, Vanderbilt University, Nashville, TN 37235 USA
}

\begin{abstract}
Measurement of the fusion excitation function for ${ }^{18} \mathrm{O}+{ }^{12} \mathrm{C}$ and ${ }^{19} \mathrm{O}+{ }^{12} \mathrm{C}$ is described. The fusion cross-section is extracted through the direct measurement of evaporation residues resulting from the fusion process. At near barrier energies, the single additional neutron present in ${ }^{19} \mathrm{O}$ results in an enhancement in the fusion cross-section by a factor of over three as compared to ${ }^{18} \mathrm{O}$.
\end{abstract}

\section{Introduction}

Measuring the fusion excitation function for an isotopic chain of projectile nuclei presents an unique opportunity to examine the character of neutron-rich matter. For a given element, with increasing neutron number the neutron density distribution usually extends further out while the proton distribution remains largely unaffected. Hence, the repulsive Coulomb potential is largely unchanged while the attractive nuclear potential changes. As fusion at near barrier energies is sensitive to the interplay between the repulsive Coulomb and attractive nuclear potentials, the comparison of the fusion excitation function for isotopically related projectiles provides a sensitive probe of the change in the attractive nuclear potential. This change in the attractive potential can be related to changes in both the structure and dynamics of the neutron density distribution as the number of neutrons increases.

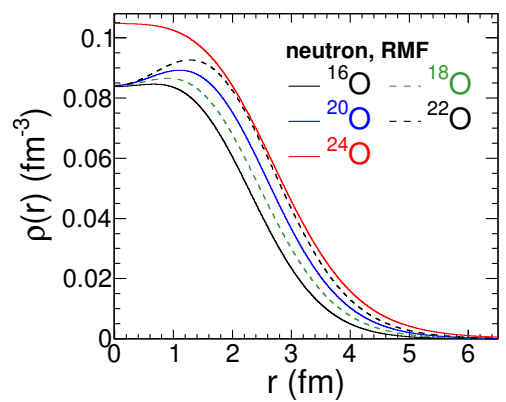

Figure 1. Neutron-density distribution of oxygen isotopes calculated within a relativistic mean field theory (RMF).

\footnotetext{
^e-mail: desouza@indiana.edu
}

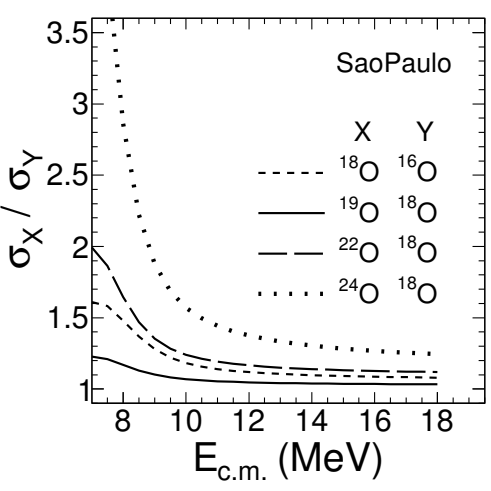

Figure 2. Relative cross-section for Oxygen isotopes using a simple barrier penetration model with the RMF density distributions.

Presented in Fig. 1 are the neutron density distributions for oxygen isotopes calculated within the context of a relativistic mean field theory $[1,2]$. As expected, with increasing neutron number the tail of the density distribution extends further out. It is perhaps surprising however that the tail of the neutron density distribution for ${ }^{22} \mathrm{O}$ is so close to that of the drip-line nucleus ${ }^{24} \mathrm{O}$. This observation is particularly noteworthy as beams of ${ }^{20,21} \mathrm{O}$ are presently available at GANIL and a beam of ${ }^{22} \mathrm{O}$ is anticipated in the near future. In this paper, we describe the first step towards the systematic measurement of the fusion excitation functions of the oxygen isotopes namely fusion in ${ }^{18} \mathrm{O}+{ }^{12} \mathrm{C}$ and ${ }^{19} \mathrm{O}+{ }^{12} \mathrm{C}$.

An extended neutron distribution could impact fusion both through its static spatial extent as well as through its dynamics (e.g. polarization effects). The influence of the static contribution can be estimated by using a onedimensional barrier penetration model such as the Sao- 
Paulo model [3]. For the density distributions presented in Fig. 1, we have calculated the relative fusion crosssections for several oxygen isotopes and present the results in Fig. 2. Evident in the figure is the fact that at all energies as the neutron-richness increases the fusion cross-section increases. At energies well above the barrier $\left(\mathrm{E}_{c . m .} \sim 8\right.$ $\mathrm{MeV}$ ) this enhancement is relatively constant and can be considered geometric. Near and below the barrier however, the enhancement increases rapidly with decreasing incident energy. In this energy domain the enhancement reflects the increased importance of the nuclear potential due to the neutron-skin. It should be emphasized that as this is a purely static calculation, the inclusion of fusion dynamics could result in an enhancement larger than that depicted in Fig. 2.

In order to measure the fusion excitation function for ${ }^{18} \mathrm{O}+{ }^{12} \mathrm{C}$ and ${ }^{19} \mathrm{O}+{ }^{12} \mathrm{C}$ two separate experiments were performed at the John D. Fox accelerator laboratory at Florida State University. In the initial experiment we measured the fusion excitation function for ${ }^{18} \mathrm{O}+{ }^{12} \mathrm{C}$, extending the measured cross-section down to the sub $1 \mathrm{mb}$ level, a factor of 30 lower than previously measured. Having established the technique, we subsequently measured the fusion excitation function for ${ }^{19} \mathrm{O}+{ }^{12} \mathrm{C}$. The experimental details of both measurements are summarized below.

\section{The ${ }^{18} \mathrm{O}+{ }^{12} \mathrm{C}$ experiment}

\subsection{Experimental setup}

The experimental setup for this experiment consisted of two ExB microchannel plate detectors and two annular silicon detectors as depicted in Fig. 3. The beam first passes through a ExB microchannel plate detector, designated $\mathrm{MCP}_{\mathrm{US}}$, situated approximately $1.3 \mathrm{~m}$ upstream of the target position. For each ion traversing this detector a fast timing signal is generated. The beam subsequently encounters a second ExB microchannel plate detector, designated $\mathrm{MCP}_{\mathrm{TGT}}$. The $100 \mu \mathrm{g} / \mathrm{cm}^{2}$ thick carbon foil of $\mathrm{MCP}_{\mathrm{TGT}}$ is dual function. Not only does it serve as a secondary emission for the detector, but it also serves as the target for the experiment. The coincidence of these two ExB MCP detectors with the appropriate time-of-flight provided the incident beam count. The typical intensity of the ${ }^{18} \mathrm{O}$ beam incident on the target was $\sim 2 \times 10^{5}$ ions/s.

\subsection{Measuring the fusion products}

Fusion of a ${ }^{18} \mathrm{O}$ nucleus in the beam together with $a{ }^{12} \mathrm{C}$ nucleus in the target foil results in the production of an excited ${ }^{30} \mathrm{Si}$ nucleus. For collisions near the Coulomb barrier the excitation of the fusion product is relatively modest, $\mathrm{E}^{*} \approx 35 \mathrm{MeV}$. De-excitation of this fusion product by evaporation of a few neutrons, protons, and $\alpha$ particles results in an evaporation residue (ER). Statistical model calculations [4] indicate that for a ${ }^{30} \mathrm{Si}$ compound nucleus, the nuclei ${ }^{29} \mathrm{Si},{ }^{28} \mathrm{Si},{ }^{28} \mathrm{Al},{ }^{27} \mathrm{Al}$, and ${ }^{25} \mathrm{Mg}$ account for the bulk of the ERs. Emission of the light particles deflects the ER from the beam direction allowing their detection and identification in two annular silicon detectors, designated $\mathrm{T} 2$ and $\mathrm{T} 3$, that are situated downstream of the $\mathrm{MCP}_{\text {TGT. }}$ These detectors subtend the angular range $3.5^{\circ}$ $<\theta_{l a b}<25^{\circ}$ allowing detection of the majority of the ERs produced [5]. By measuring the time-of-flight of particles between the $\mathrm{MCP}_{\mathrm{TGT}}$ detector and the silicon detectors [6] together with the energy deposit in the Si detector, evaporation residues are distinguished from scattered beam, as well as emitted light particles. By utilizing the measured energy deposit and time-of-flight, the mass of the ion can be calculated providing clear separation of ERs from the incident beam [7].

\subsection{Fusion excitation function for ${ }^{18} \mathrm{O}+{ }^{12} \mathrm{C}$}

The fusion cross-section is extracted by summing the total number of evaporation residues observed. Comparison of this yield with the number of incident ${ }^{18} \mathrm{O}$ ions while accounting for the target thickness and the geometric efficiency of the experimental setup yields the absolute fusion cross-section [7]. The measured excitation function is displayed in Fig. 4a together with previously published results [9-11]. Vertical error bars on the new data reflect both the statistical uncertainties as well as a $2 \%$ systematic error associated with the analysis. Horizontal error bars represent the uncertainty in whether the fusion occurs at the front or back of the target foil. While prior measurements using the direct measurement of evaporation residues only measured the fusion cross-section down to the $25 \mathrm{mb}$ level [9], in this work the fusion cross-section is measured down to the $820 \mu \mathrm{b}$ level, a factor of approximately 30 lower in cross-section. In the energy region where the present data overlaps with published data, overall agreement of the cross-sections is good, close to the statistical uncertainties. This overall agreement indicates both that our approach in extracting the fusion crosssection is sound and that there are no significant uncertainties in the values of the target thickness or detector efficiency. Closer comparison of the present dataset with the data of Ref. [9] indicates that the presently measured cross-sections are approximately 3-5\% lower for $\mathrm{E}_{\mathrm{c} . \mathrm{m} .} \geq$ $10 \mathrm{MeV}$. This is within the statistical uncertainties of the data reported by Eyal et al. In addition, the present data are higher in their statistical quality.

We have compared the experimental fusion excitation function with the predictions of a microscopic model. Over the past several years, the density constrained TDHF (DC-TDHF) method for calculating heavy-ion potentials [12] has been employed to calculate heavy-ion fusion cross-sections with remarkable success [13, 14]. While most applications have been for systems involving heavy nuclei, recently the theory was used to study above and below barrier fusion cross-sections for lighter systems, specifically for reactions involving various isotopes of $\mathrm{O}+\mathrm{O}$ and $\mathrm{O}+\mathrm{C}[15,16]$. One general characteristic of TDHF and DC-TDHF calculations for light systems is that the fusion cross-section at energies well above the barrier are usually overestimated [17, 18], whereas an excellent agreement is found for sub-barrier cross-sections [15]. 


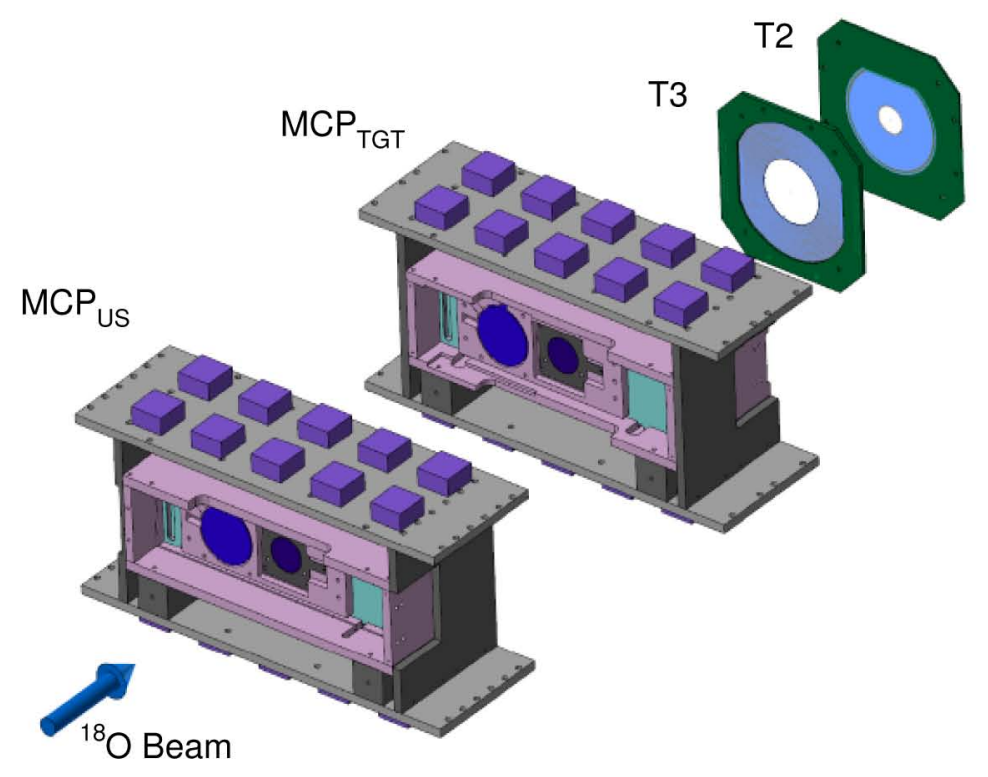

Figure 3. Experimental setup utilized to directly measure the evaporation residues and extract the fusion cross-section

Comparison of the experimental fusion excitation function with the DC-TDHF microscopic model is presented in Fig. 4a. Overall comparison of the experimental cross-sections with the DC-TDHF calculations indicate that for energies $\gtrsim 7 \mathrm{MeV}$ the experimental cross-sections (symbols) are lower than the theoretical predictions (solid line). In order to facilitate a quantitative comparison of the experimental excitation function with the theoretical predictions, we have fit the experimental cross-sections with a functional form [19] that describes the penetration of an inverted parabolic barrier. The values of the fit parameters are presented in Table 1.

$$
\sigma=\frac{R_{C}^{2}}{2 E} \hbar \omega \cdot \ln \left\{1+\exp \left[\frac{2 \pi}{\hbar \omega}\left(E-V_{C}\right)\right]\right\}
$$

where $E$ is the incident energy, $V_{C}$ is the barrier height, $R_{C}$ is the radius of interaction, and $\hbar \omega$ is the barrier curvature.

Shown in Fig. $4 b$ is the ratio of the fit of the experimentally measured cross-sections to the DC-TDHF calculations. For energies $7.5 \mathrm{MeV}<\mathrm{E}_{\text {c.m. }}<14 \mathrm{MeV}$, the ratio $\sigma_{\text {Experiment }} / \sigma_{\mathrm{DC}-\mathrm{TDHF}}$ is $\approx 0.75$ and is relatively constant with respect to energy. As the incident energy decreases below $7.5 \mathrm{MeV}$, the ratio increases reaching a value of over 10 at the lowest energy measured, $\mathrm{E}_{\text {c.m. }} \approx 5 \mathrm{MeV}$. The fact that the ratio is only $\approx 0.75$ at energies above the barrier can be understood as due to the presence of breakup reactions in this energy range. With decreasing incident energy, the role of breakup reactions diminshes hence the ability of the DC-TDHF model to describe fusion is expected to improve. We therefore focus our attention on the comparison of the model and experiment in the subbarrier region. The key feature in the ratio is therefore its change with decreasing incident energy in the sub-barrier domain, specifically its increase from a value smaller than unity to a value larger than unity. This trend emphasizes that the experimental and theoretical excitation functions

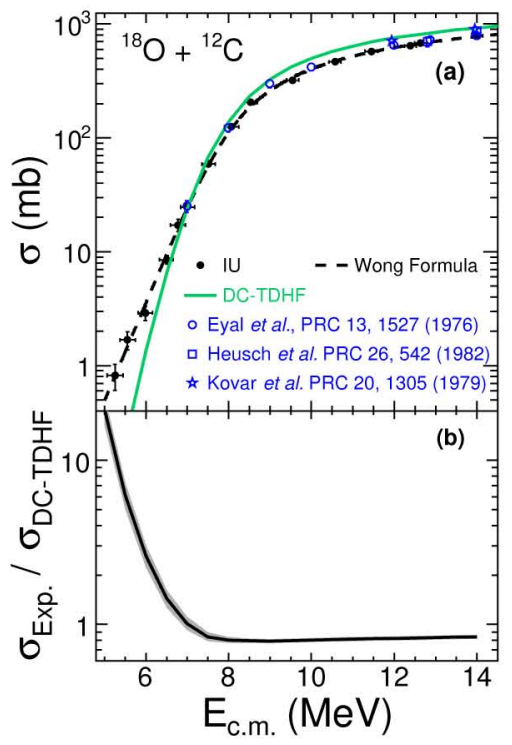

Figure 4. Comparison of the measured cross-section for ${ }^{18} \mathrm{O}+{ }^{12} \mathrm{C}$ with the predictions of the DC-TDHF model.

have different shapes with the experimental cross-section falling more slowly with decreasing incident energy than is theoretically predicted by the DC-TDHF model. This enhancement of the experimental fusion cross-sections relative to the DC-TDHF predictions is a factor of $\approx 10$ as the incident energy decreases from $E_{c . m .}=7 \mathrm{MeV}$ to $E_{c . m .}=$ $5 \mathrm{MeV}$. We have assessed the impact of the experimental uncertainties on the ratio presented and display the result as a shaded band in Fig. 4b. The trends exhibited by the ratio are significantly larger than the magnitude of the uncertainties.

The fact that the experimental fusion cross-sections decrease more slowly with decreasing energy than the calculated cross-sections can be interpreted as a larger tun- 
neling probability for the experimental data as compared to the theoretical calculations. This enhanced tunneling probability can be associated with a narrower, lower barrier. The underlying reason that the barrier determined from the experimental data is weaker than in the model is presently unclear.

\section{The ${ }^{19} \mathrm{O}+{ }^{12} \mathrm{C}$ experiment}

\subsection{Producing and Chararacterizing a ${ }^{19} \mathrm{O}$ beam}

A beam of ${ }^{18} \mathrm{O}$ ions at an energy of $80.7 \mathrm{MeV}$ was used to bombard a deuterium gas cell at a pressure of 350 torr to produce the ${ }^{19} \mathrm{O}$ beam. To increase the gas density, the gas cell was cooled to a temperature of $77 \mathrm{~K}$. Ions of ${ }^{19} \mathrm{O}$ produced via a $(\mathrm{d}, \mathrm{p})$ reaction were separated from the incident beam by the electromagnetic spectrometer RESOLUT [20]. Despite the rejection of most of the unreacted beam by RESOLUT, the beam exiting the spectrometer consisted of both ${ }^{19} \mathrm{O}$ and ${ }^{18} \mathrm{O}$ ions. It was therefore necessary to identify each ion incident on the target. By accomplishing this it was possible to simultaneously measure the fusion excitation function for ${ }^{18} \mathrm{O}+{ }^{12} \mathrm{C}$ and ${ }^{19} \mathrm{O}+{ }^{12} \mathrm{C}$ which provided an important consistency check. Comparison of the ${ }^{18} \mathrm{O}+{ }^{12} \mathrm{C}$ with the prior high statistics measurement of ${ }^{18} \mathrm{O}+{ }^{12} \mathrm{C}$ demonstrated that there were no systematic differences between the two measurements. This dual measurement thus provided confidence that any observed fusion enhancement was a robust signal.

\subsection{Experimental setup}

Although the experimental setup for the ${ }^{19} \mathrm{O}$ beam was largely the same as in the prior experiment, a few changes were made to handle the radioactive beam. In order to identify beam particles, the energy deposit $(\Delta \mathrm{E})$ and timeof-flight (TOF) of each particle was measured prior to the target. After exiting RESOLUT particles traversed a thin foil $(0.5 \mu \mathrm{m}$ thick aluminized mylar) which served as the electron emission foil for an MCP detector. Approximately $3.5 \mathrm{~m}$ downstream of this thin foil the oxygen ions passed through a compact ionization detector (CID) depositing an energy, $\Delta \mathrm{E}$. CID served two roles: to help identify the ion as well as to reduce its energy. While the production of ${ }^{19} \mathrm{O}$ is favored at higher energies, the measurement of the fusion cross-section at near barrier energies requires reducing the energy of the radioactive ion after its production. To perform the excitation function measurement, the energy of the incident beam was decreased by adjusting the gas pressure in CID. Upon exiting CID the ions were incident on a $105 \mu \mathrm{g} / \mathrm{cm}^{2}$ carbon foil. This carbon foil, as in the prior experiment, served both as a secondary electron emission foil for the target microchannel plate detector $\left(\mathrm{MCP}_{\mathrm{TGT}}\right)$ and as the target for the fusion experiment [8]. To measure the energy distribution of ${ }^{19} \mathrm{O}$ and ${ }^{18} \mathrm{O}$ ions incident on the target, a surface barrier, silicon detector was periodically inserted into the beam path just prior to the target.

The timing signals from both microchannel plate detectors together with the energy deposit in the ionization chamber allowed identification of ions in the beam through measurement of the $\triangle \mathrm{E}$-TOF. Ions of ${ }^{19} \mathrm{O}^{7+},{ }^{18} \mathrm{O}^{7+}$, and ${ }^{18} \mathrm{O}^{6+}$ are clearly identified with ${ }^{19} \mathrm{O}$ ions corresponding to approximately $31 \%$ of the beam intensity. The two charge states of ${ }^{18} \mathrm{O}$ contributed to approximately $50 \%$ of the beam intensity on target. The intensity of the ${ }^{19} \mathrm{O}$ beam incident on the target was $1.5-4 \times 10^{3}$ ions/s.

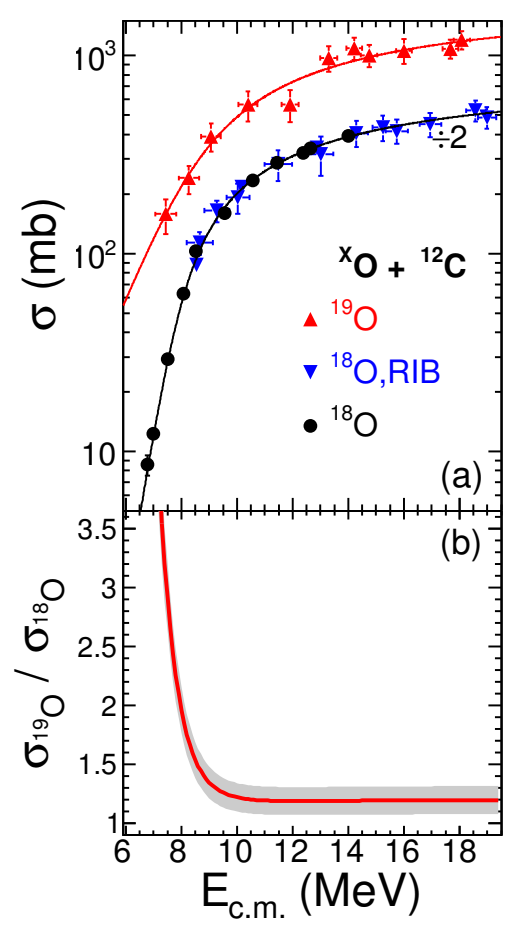

Figure 5. Comparison of the measured cross-section for ${ }^{18} \mathrm{O}+$ ${ }^{12} \mathrm{C}$ and ${ }^{19} \mathrm{O}+{ }^{12} \mathrm{C}$. The cross-sections for the ${ }^{18} \mathrm{O}$ reaction have been scaled by a factor of two for clarity.

\subsection{Fusion excitation function for ${ }^{19} \mathrm{O}+{ }^{12} \mathrm{C}$}

The same general trend is observed for both of the excitation functions depicted in Fig. 5a. With decreasing incident energy the cross-section decreases as expected for a barrier controlled process. At essentially all energies measured the ${ }^{19} \mathrm{O}$ data exhibits a larger fusion cross-section as compared to the ${ }^{18} \mathrm{O}$ data.

Table 1. Fit parameters for the ${ }^{x} \mathrm{O}+{ }^{12} \mathrm{C}$ fusion excitation functions. See text for details.

\begin{tabular}{|c|c|c|c|}
\hline & $V_{C}(\mathrm{MeV})$ & $R_{C}(\mathrm{fm})$ & $\hbar \omega(\mathrm{MeV})$ \\
\hline${ }^{18} \mathrm{O}+{ }^{12} \mathrm{C}$ & $7.66 \pm 0.10$ & $7.39 \pm 0.11$ & $2.90 \pm 0.18$ \\
${ }^{19} \mathrm{O}+{ }^{12} \mathrm{C}$ & $7.73 \pm 0.72$ & $8.10 \pm 0.47$ & $6.38 \pm 1.00$ \\
\hline
\end{tabular}

To quantitatively examine the differences in the two excitation functions we have fit the excitation functions with the Wong formalism of penetration of an inverted parabolic barrier [19]. The fit of the high resolution ${ }^{18} \mathrm{O}$ data is indicated as the solid black line in Fig. 5a. The solid red curve in Fig. 5a depicts the fit of the ${ }^{19} \mathrm{O}$ data. A reasonable fit of the measured fusion cross-sections is achieved in both cases. The extracted parameters for the 
${ }^{18} \mathrm{O}$ and ${ }^{19} \mathrm{O}$ reactions are summarized in Table 1 . Since the charge density distribution is essentially unchanged, it is unsurprising that the barrier height, $V_{C}$, remains essentially the same for both of the reactions examined. Moreover, as expected with increasing neutron number an increase in $R_{C}$ is observed. This increase in the radius can be viewed by calculating the quantity $R_{C} / \mathrm{A}^{1 / 3}$ where $\mathrm{A}$ is the mass number of the compound nucleus. This quantity has a value of 2.38 for the ${ }^{18} \mathrm{O}$ induced reaction, while it is 2.58 for the ${ }^{19} \mathrm{O}$ induced reaction. The most significant change in the fit parameters is a substantial increase in the magnitude of $\hbar \omega$ for the ${ }^{19} \mathrm{O}$ case corresponding to a narrower barrier, reflecting an increase in the attractive nuclear potential.

Depicted in Fig. 5b as the solid (red) line is the dependence of the measured ratio of $\sigma\left({ }^{19} \mathrm{O}\right) / \sigma\left({ }^{18} \mathrm{O}\right)$ on $\mathrm{E}_{\mathrm{c} . \mathrm{m} .}$. At energies well above the barrier $\sigma\left({ }^{19} \mathrm{O}\right) / \sigma\left({ }^{18} \mathrm{O}\right)$ is essentially flat at a value of $\approx 1.2$. As one approaches the barrier it rapidly increases to a value of approximately 3.5. Hence, the addition of a single additional neutron in ${ }^{19} \mathrm{O}$ as compared to ${ }^{18} \mathrm{O}$ results in a dramatic enhancement in the fusion cross-section at sub-barrier energies.

\section{Summary}

We have measured the fusion excitation functions for ${ }^{18} \mathrm{O}$ $+{ }^{12} \mathrm{C}$ and ${ }^{19} \mathrm{O}+{ }^{12} \mathrm{C}$ using low intensity beams. Comparison of these excitation functions indicates a significant enhancement at near barrier energies for the neutron-rich projectile. The addition of a single neutron increases the fusion cross-section by more than a factor of three at the lowest energy measured. This enhancement may reflect the increased role of neutron transfer or coupling to collective degrees of freedom. These measurements represent the first step in the measurement of the fusion excitation function for an isotopic chain of oxygen nuclei. Acquiring a systematic, high quality dataset of this type, coupled with microscopic calculations of the fusion process has considerable promise in elucidating the nature of neutron-rich nuclear matter.

\section{Acknowledgements}

The support of the staff at Florida State University's John D. Fox accelerator in providing the ${ }^{18} \mathrm{O}$ and ${ }^{19} \mathrm{O}$ beams made this experiment feasible. We gratefully acknowledge their efforts. Development of the Sao Paulo model code by Dr. Helber Dussan is gratefully acknowledged. This work was supported by the U.S. Department of Energy under Grant Nos. DE-FG02-88ER-40404 (Indiana University), DE-FG02-87ER40365 (Indiana University Nuclear Theory), DE-SC0008808 (NUCLEI SciDAC Col- laboration), DE-FG02-02ER-41220 (Florida State University), DE-SC0013847 (Vanderbilt University) and the National Science Foundation under Grant No PHY-1491574 (Florida State University). J.V. acknowledges the support of a NSF Graduate Research Fellowship under Grant No. 1342962.

\section{References}

[1] P. Ring, Prog. Part. Nucl. Phys. 37, 193 (1996)

[2] B. D. Serot and J. D. Walecka, Adv. Nucl. Phys. 16, 1 (1986)

[3] L. R. Gasques et al., Phys. Rev. C 69, 034603 (2004)

[4] N. G. Nicolis and J. R.Beene, unpublished, 1993

[5] T. K. Steinbach, PhD Thesis, Indiana University (2016)

[6] R. T. deSouza et al., Nucl. Instr. and Meth. A632, 133 (2011)

[7] T. K. Steinbach et al., Phys. Rev. C. 90, 041603(R)(2014)

[8] T. K. Steinbach et al., Nucl. Instr. and Meth. A743, 5 (2014)

[9] Y. Eyal and M. Beckerman and R. Checkhik and Z. Fraenkel and H. Stocker, Phys. Rev. C 131527 (1976)

[10] D. Kovar, D.F. Geesaman, T.H. Braid, Y. Eisen, W. Henning, T.R. Ophel, M. Paul, K.E. Rehm, S.J. Sanders, P. Sperr, J.P. Schiffer, S.L. Tabor, S. Vigdor, B. Zeidman and F.W. Prosser Jr., Phys. Rev. C 20, 1305 (1979)

[11] B. Heusch, C. Beck, J.P. Coffin, P. Engelstein, R. M. Freeman, G. Guillaume, F. Haas, P. Wagner, Phys. Rev. C 26, 542 (1982)

[12] A. S. Umar and V. E.Oberacker, Phys. Rev. C 74 021601(R) (2006)

[13] R. Keser and A. S. Umar and V. E.Oberacker, Phys. Rev. C 85044606 (2012)

[14] Back, B. B., Esbensen, H., Jiang, C. L. and Rehm, K. $\mathrm{E}$., Recent developments in heavy-ion fusion reactions Rev. Mod. Phys. 86, 317 (2014)

[15] A. S. Umar, V. E.Oberacker and C. J. Horowitz, Phys. Rev. C 85055801 (2012)

[16] R. T. deSouza, S. Hudan and V. E. Oberacker and A. S. Umar", Phys. Rev. C 88, 014602 (2013)

[17] C. Simenel, R. Keser, A. S. Umar and V. E. Oberacker, Phys. Rev. C, 88, 024617 (2013)

[18] A. S. Umar, C. Simenel and V. E. Oberacker, Phys. Rev. C 89, 034611 (2014)

[19] C. Y. Wong, Phys. Rev. Lett.31, 766 (1973)

[20] I. Wiedenhöver et al., Fifth International Conference on Fission and Properties of Neutron-rich Nuclei, Eds. J.H. Hamilton and A.V. Ramayya, World Scientific, Sanibel Island, FL 2012 